\title{
Incidencia de tuberculosis en pacientes que reciben fármacos anti-TNF $\alpha$
}

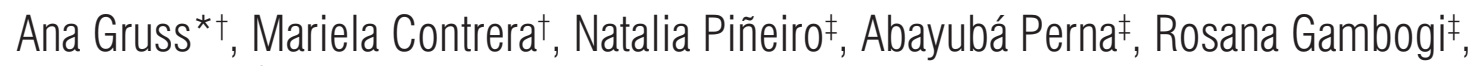
Alicia Alemán§, Fernando Correa ${ }^{\ddagger}$, Henri Albornoz ${ }^{\ddagger}$

\section{Resumen}

Introducción: la tuberculosis (TB) es una complicación frecuente del uso de fármacos anti-TNF $\alpha$. Ocurre por reactivación de una infección latente o por progresión de una infección reciente.

Objetivos: conocer la incidencia de TB en la población que recibió fármacos anti-TNF $\alpha$, analizar las formas de presentación y la realización de pesquisa de infección latente previo al inicio del tratamiento.

Método: estudio de cohorte retrospectiva. Se incluyeron los pacientes que recibieron fármacos anti-TNF $\alpha$ entre 2010 y 2016. Los datos se obtuvieron de los sistemas informáticos del Fondo Nacional de Recursos y del Programa Nacional de Tuberculosis. Se calculó la incidencia de TB y se describieron los casos que desarrollaron TB.

Resultados: se incluyeron 991 tratamientos para un total de 980 pacientes. Se reportaron nueve casos de TB. La incidencia global fue de 419,9 (IC 95\% 191,9-591,2) por 100.000 personas/año. Solo hubo casos de TB en pacientes tratados con adalimumab. El cribado de infección tuberculosa latente (ITBL) previo al inicio del fármaco fue heterogéneo y predominaron las formas de TB diseminadas (6/9) sobre la afectación pulmonar aislada (3/9). En todos los casos se suspendió el anti-TNF $\alpha$ al diagnóstico de TB y en ningún caso se retomó.

Conclusiones: la incidencia de TB en la población de pacientes bajo tratamiento con anti-TNF $\alpha$ fue 16,5 veces mayor que en la población general. Predominaron las formas de TB diseminadas y se dieron casos en sujetos que habían recibido tratamiento de ITBL previo al inicio del fármaco, sugiriendo que el riesgo persiste mientras exista exposición a éste.

Palabras clave: Tuberculosis

Tuberculosis latente

Anti-TNF $\alpha$

Factor de necrosis tumoral alfa

Adalimumab

Etanercept

Infliximab

Golimumab
Key words: $\quad$ Tuberculosis

Latent tuberculosis

Anti-TNF $\alpha$

Tumor necrosis factor-alpha

Adalimumab

Etanercept

Infliximab

Golimumabe

* Cátedra de Neumología, Instituto del Tórax, Hospital Maciel.

† Comisión Honoraria de Lucha Antituberculosa y Enfermedades Prevalentes.

‡ Fondo Nacional de Recursos.

$\S$ Departamento de Medicina Preventiva y Social, Facultad de Medicina, Universidad de la República, Uruguay.

Correspondencia: Dra. Ana Inés Gruss. Hospital Maciel, Cátedra de Neumología, 25 de Mayo 174, CP 11000, Montevideo, Uruguay. Correo electrónico: anainesgruss@gmail.com

Los autores declaran no tener conflictos de interés.

Aprobado por el Comité de Ética del Hospital Español.

Recibido: 26/5/19

Aprobado: 5/8/19 


\section{Introducción}

La tuberculosis (TB) constituye un problema prioritario para la salud pública mundial ${ }^{(1)}$. Actualmente, representa la novena causa de muerte en el mundo y la primera por enfermedades infecciosas, estando por encima del VIH/SIDA ${ }^{(1)}$. En el año 2016, se estima que 10,4 millones de personas en el mundo contrajeron TB, lo que equivale a una incidencia global de 142 casos cada 100.000 habitantes, registrándose 1,67 millones de muertes ${ }^{(1)}$.

Se estima que un tercio de la población mundial estaría infectada por $M$. tuberculosis y que solo $5 \%$ a $10 \%$ podría desarrollar la enfermedad a lo largo de su vida. Sin embargo, existen condiciones que aumentan el riesgo de desarrollarla, principalmente las vinculadas a inmunodepresión por enfermedades (VIH, enfermedades autoinmunes, hematooncológicas, etcétera), o por administración de drogas inmunosupresoras ${ }^{(2-4)}$.

En el grupo de drogas inmunosupresoras utilizadas en la actualidad se destacan los fármacos anti-TNF $\alpha$, los que han demostrado ser altamente beneficiosos en el tratamiento de enfermedades inflamatorias crónicas, como la artritis reumatoidea (AR), espondilitis anquilosante (AE), enfermedad de Crohn (EC) y colitis ulcerosa crónica (CUC). Las principales complicaciones de estos fármacos son las infecciones y en particular la TB. La TB se podría desarrollar a partir de la reactivación de una infección tuberculosa latente (ITBL) en un individuo que ha tenido contacto previamente con $M$. tuberculosis, o por progresión de una infección adquirida en forma reciente ${ }^{(5)}$.

Se resumen los principales mecanismos que determinan la asociación entre el uso de anti-TNF $\alpha$ y TB.

\section{TNF $\alpha$ y TB}

EL TNF $\alpha$ es un componente fundamental en la respuesta inmune del huésped frente a $M$. tuberculosis. Su acción sinérgica con el IFN gama, mediador central de linfocitos Th1, contribuye a la activación de los macrófagos y posterior diferenciación en células epiteloides, lo cual determina la constitución del granuloma. La ausencia de TNF $\alpha$ produce la disrupción de éste con la posibilidad de progresión de la infección y eventual diseminación.

\section{Anti-TNF $\alpha$ y TB}

Los fármacos anti-TNFa se diferencian por sus mecanismos de acción: 1) anti-TNF $\alpha$ solubles que interactúan principalmente con TNF $\alpha$ soluble (TNFR1): adalimumab, infliximab, golimumab, certolizumab y 2) anti-TNF $\alpha$ que interactúan con el receptor de TNF ligado a la membrana (TNFR2): etanercept. El TNFR1 es esencial para la formación del granuloma, a diferencia de TNFR2, que tiene una acción menor, lo cual explicaría las diferentes acciones de estos fármacos en relación con el granuloma tuberculoso (disrupción y diseminación del $M$. tuberculosis) y, por lo tanto, al riesgo de reactivación de una $\operatorname{ITBL}^{(6)}$.

En Uruguay, el Fondo Nacional de Recursos (FNR) como institución parte del Sistema Nacional Integrado de Salud, brinda cobertura financiera para tratamientos con fármacos biológicos, entre ellos los anti-TNF $\alpha$. En el año 2010 comenzó la cobertura para AR y posteriormente se amplió para EA, EC y CUC, lo cual ha determinado una utilización progresiva de dichos fármacos en nuestro medio.

Por otra parte, se ha observado un cambio en la situación epidemiológica de la TB en Uruguay, ocurriendo un aumento progresivo de la incidencia, la cual alcanzó 25,7/100.000 habitantes para el año 2016. Los casos se distribuyen en forma diferente según los departamentos, siendo Montevideo el que registra la incidencia mayor: 38,6/100.000 habitantes. En relación con los grupos etarios, se concentra en edades jóvenes (15 a 54 años), lo que traduce una alta transmisibilidad de la infección en la comunidad $^{(7)}$.

El objetivo principal del estudio fue conocer la incidencia de TB en la población que inició tratamiento con fármacos anti-TNF $\alpha$ (adalimumab, etanercept, golimumab e infliximab) bajo la cobertura del FNR en el período entre 2010 y 2016. Los objetivos secundarios fueron analizar las formas de presentación de la TB y la pesquisa de infección tuberculosa latente en esta población previa al inicio de dichos tratamientos.

\section{Material y método}

Se realizó un estudio de cohorte retrospectiva que incluyó los tratamientos autorizados, desde el inicio de la cobertura (AR marzo 2010, EC marzo 2010, EA octubre 2013, CUC diciembre 2015) hasta el 31 de diciembre de 2016. Los datos se obtuvieron de los sistemas informáticos del FNR y del Programa Nacional de Tuberculosis (PNT). Las variables estudiadas en relación con los agentes anti-TNF $\alpha$ fueron: fecha de inicio del tratamiento y tipo de fármaco utilizado: adalimumab, golimumab, etanercept o infliximab. Aquellos pacientes que iniciaron tratamiento con anti-TNF $\alpha$ y que en algún momento cambiaron por otro fármaco no anti-TNF $\alpha$, se los censuró a la fecha del cambio. Se estudiaron variables demográficas de la población y en relación con la TB: mediana de seguimiento, forma de presentación clínico-radiológica, bacteriología, complicaciones durante el tratamiento de la TB, resultado de tratamiento, métodos diagnósticos utilizados para el cribado de ITBL y tratamiento de ésta. Se tomó como caso de TB a aquel paciente en el que el médico tratante decide iniciar trata- 
Tabla 1. Características demográficas de la población estudiada según enfermedad inflamatoria sistémica.

\begin{tabular}{|c|c|c|c|c|}
\hline & $\begin{array}{c}A R \\
n=648 \\
t=653\end{array}$ & $\begin{array}{l}\text { CUC } \\
n=15 \\
t=15\end{array}$ & $\begin{array}{c}E A \\
n=252 \\
t=254\end{array}$ & $\begin{array}{c}E C \\
n=65 \\
t=69\end{array}$ \\
\hline Edad años (media) & 48,6 & 48,8 & 42,5 & 34,9 \\
\hline Sexo femenino (\%) & 83,8 & 53,3 & 43,7 & 53,6 \\
\hline Procedencia Montevideo (\%) & 48,9 & 53,3 & 53,6 & 69,2 \\
\hline
\end{tabular}

miento anti-TB, ya sea por sospecha clínica, confirmación bacteriológica, adenosín deaminasa (ADA) positiva o histopatología compatible, o ambas. Los estudios para el diagnóstico de TB fueron: baciloscopía con técnica de Zihel Neelsen o auramina rodamina, cultivos en medios líquidos y sólidos, histopatología sugestiva de TB y $\mathrm{ADA}$ en líquido cefalorraquídeo (LCR) o alguna serosa positiva para TB. Para ITBL, los tests diagnósticos que se usaron fueron: prueba de Mantoux o PPD e Interferon Gamma Release Assays (IGRA).

La tasa de incidencia de TB se calculó considerando los nuevos casos en el período estudiado, dividido la sumatoria de los tiempos individuales de exposición, y se calculó su respectivo intervalo de confianza a 95\%.

Para el cálculo de las respectivas medianas de seguimiento se excluyeron aquellos pacientes que tuvieron el evento (TB).

Para el análisis estadístico se utilizó el software Stata 15.0 y WinPepi 4.0 y R 3.0.3

\section{Resultados}

\section{Características de la población estudiada}

Se incluyeron 991 tratamientos para un total de 980 pacientes, 653 con AR (648 pacientes), 254 con EA ( 252 pacientes), 69 con EC ( 65 pacientes) y 15 con CUC ( 15 pacientes). En la tabla 1 se describen las características demográficas de la población según la patología de base: AR, CUC, EA o EC.

La mediana de seguimiento global fue de 22,5 meses. Para AR 24,7 meses; EC 23,7 meses; EA 20,3 meses, y el menor seguimiento correspondió a los pacientes con CUC: 2,9 meses.

\section{Incidencia de tuberculosis en pacientes bajo tratamiento con anti-TNF $\alpha$}

La ocurrencia de enfermedad tuberculosa fue de 9 casos en el período considerado, 6 de ellos fueron en pacien-
Tabla 2. Tasa de incidencia global de tuberculosis, procedencia y separado por patologías (para 100.000 personas /año).

\begin{tabular}{lc}
\hline Global & 419,9 (IC 95\% 191,9-796,8) \\
Tasa Montevideo & 602,6 (IC 95\% 242,3-1241,7) \\
Tasa interior & 203,6 (IC 95\% 24,6-735,4) \\
AR & 385,7 (IC 95\% 141,5-839,4) \\
EC & 1256,6 (IC 95\% 152-4539,6) \\
CUC & 0 \\
EA & 234,9 (IC 95\% 5,8-1308,7) \\
\hline & \\
AR: artritis reumatoidea; CUC: colitis ulcerosa crónica; EA: es- \\
pondilitis anquilosante; EC: enfermedad de Crohn.
\end{tabular}

tes con AR, 2 con EC, 1 con EA, y no se detectaron casos en pacientes con CUC. La incidencia global de TB para la población estudiada fue de 419,9 (IC 95\% 191,9 - 591,2) por 100.000 personas/año, 16,5 veces mayor que para la población general. En Montevideo la incidencia fue de 602,6 (IC 95\% 242,3 - 1241,7) y en el interior 203,6 (IC 95\% 24,6- 735,4) (tabla 2). La incidencia fue mayor en los pacientes con EC (1256,6 IC 95\% 152-4539,6), luego AR (385,7 IC 95\% 141,5-839,4), EA (234,9 IC 95\% 5,8-1308,7), no registrándose casos en pacientes con CUC.

La incidencia acumulada global de enfermedad tuberculosa se muestra en la figura 1 y la incidencia por patologías en la figura 2. En cuanto a la presentación temporal de los casos, la mediana de tiempo entre el inicio del fármaco y el diagnóstico de TB fue menor o igual a 15 meses. Definiendo casos de TB de presentación precoz ( $=15$ meses) y tardía ( $>15$ meses) con un rango de 4 - 44 meses (tabla 4). Cuando analizamos por patolo- 


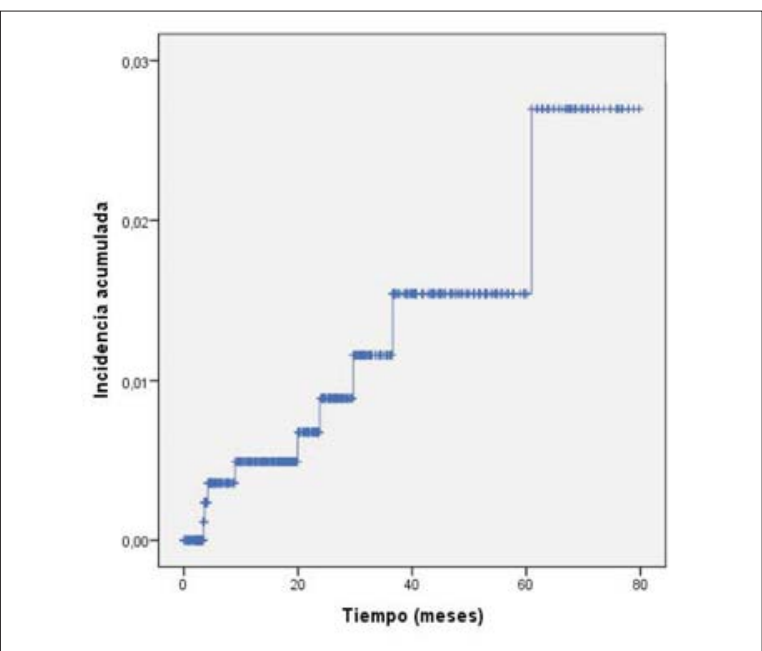

Figura 1. Incidencia acumulada global de TB en pacientes con enfermedades inflamatorias crónicas que reciben tratamiento con agentes anti-TNF $\alpha$.

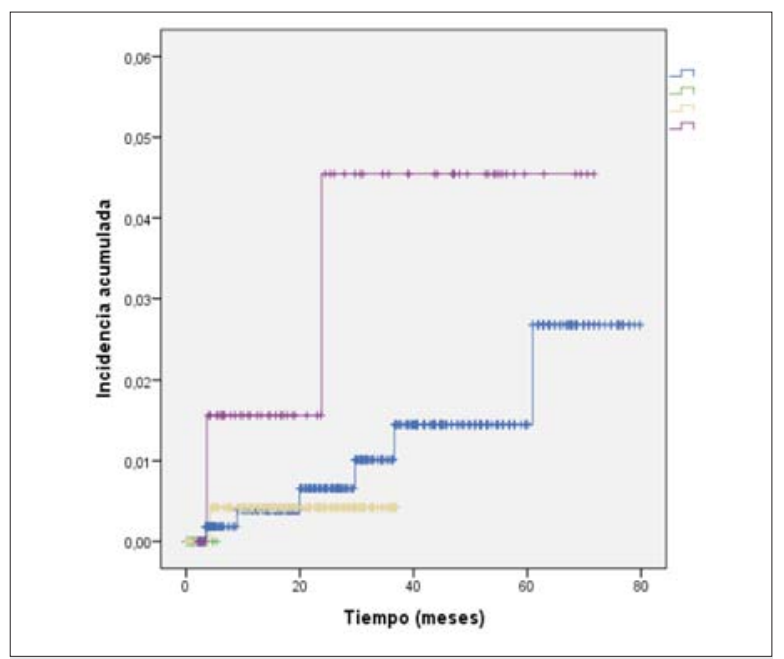

Figura 2. Incidencia acumulada de TB en pacientes con enfermedades inflamatorias crónicas que reciben tratamiento con agentes anti-TNF $\alpha$ según patología de base. AR: artritis reumatoidea; CUC: colitis ulcerosa crónica; EA: espondilitis anquilosante; EC: enfermedad de Crohn.

Tabla 3. Incidencia de enfermedad tuberculosa según fármaco.

\begin{tabular}{lcccc}
\hline \multicolumn{1}{c}{ Fármaco } & $N(\%)$ & Casos de TB & Persona/año & $\begin{array}{c}\text { Incidencia por 100.000 } \\
\text { personas año (IC 95\%) }\end{array}$ \\
\hline Infliximab & $62(6,0)$ & 0 & 137 & - \\
Golimumab & $3(0,3)$ & 0 & 0,5 & - \\
Etanercept & $221(21,3)$ & 0 & 470,9 & - \\
Adalimumab & $702(67,6)$ & 9 & 1535,2 & $586,2(268-1112,9)$ \\
Total & $988(100)$ & 9 & 2143,6 & $419,9(191,9-796,8)$ \\
\hline
\end{tabular}

gía, se observa que los casos más tardíos fueron en AR, mientras que en EA y EC ocurrieron en los primeros dos años de iniciado el fármaco.

En la tabla 3 se muestra la incidencia de TB según agente anti-TNF $\alpha$ recibido. En el período estudiado hubo $702(67 \%)$ tratamientos con adalimumab, 221 $(21,3 \%)$ con etanercept, $3(0,3 \%)$ con golimumab y 62 $(6 \%)$ con infliximab. Solo se registraron casos de TB en pacientes tratados con adalimumab, constituyendo una incidencia de 586,2 (IC 95\% 268-1112,9) por 100.000 personas/año.

\section{Características de la tuberculosis en pacientes bajo tratamiento con anti-TNF $\alpha$}

En la tabla 4 se describe, para cada caso, el screening de ITBL, el tratamiento de ITBL y las formas de pre- sentación de la TB. El screening de ITBL previo al inicio del agente biológico fue heterogéneo, realizando PPD IGRA, o ambos. En cuanto al tratamiento de la ITBL, cuatro casos recibieron INH por seis meses. Con respecto a la forma de $\mathrm{TB}$, predominaron las formas extrapulmonares o diseminadas (6/9) sobre la afectación pulmonar aislada (3/9). En todos los casos se suspendió el anti-TNF $\alpha$ al diagnosticar la TB y en ninguno de los casos se retomó.

\section{Discusión}

Nuestro estudio demostró una incidencia de TB 16 veces mayor en pacientes que recibieron anti-TNF $\alpha$ comparando con la población general. La presentación clínica fue variada así como la aparición temporal de los casos en relación con el inicio del fármaco. La presenta- 
Tabla 4. Resumen de los casos de tuberculosis desarrollados durante el tratamiento con fármacos anti-TNF $\alpha$.

\begin{tabular}{|c|c|c|c|c|c|c|c|c|c|}
\hline Casos & 1 & 2 & 3 & 4 & 5 & 6 & 7 & 8 & 9 \\
\hline $\begin{array}{l}\text { Enfermedad } \\
\text { inflamatoria }\end{array}$ & AR & $A R$ & AR & AR & AR & AR & EC & EC & EA \\
\hline $\mathrm{PPD}_{1}(\mathrm{~mm})$ & 3 & 0 & 0 & 20 & NR & 0 & 2 & 0 & NR \\
\hline $\mathrm{PPD}_{2}(\mathrm{~mm})$ & $N^{2}$ & 0 & 0 & $\mathrm{NC}^{3}$ & $\mathrm{NC}$ & 0 & NR & 0 & NC \\
\hline IGRA & Indet. & NR & NR & NR & Neg & NR & NR & NR & Neg \\
\hline Tratamiento ITBL & Sí/INH 6 ms & No & No & Si/lNH 6 ms & Sí /INH 6 ms & No & Sí/INH $6 \mathrm{~ms}$ & No & No \\
\hline Forma TB & Pulmonar & $\begin{array}{c}\text { Miliar/ } \\
\text { Ganglionar } \\
\text { /Meníngea }\end{array}$ & $\begin{array}{l}\text { Pulmonar/ } \\
\text { Ganglionar }\end{array}$ & Pulmonar & $\begin{array}{l}\text { Pulmonar/ } \\
\text { Ganglionar }\end{array}$ & Pulmonar & $\begin{array}{l}\text { Pulmonar/ } \\
\text { Ganglionar }\end{array}$ & Peritoneal & Pleural \\
\hline $\begin{array}{l}\text { Tiempo aparición } \\
\text { TB (meses) }\end{array}$ & 13 & 8 & 36 & 28 & 44 & 4 & 19 & 4 & 15 \\
\hline $\begin{array}{l}\text { Complicaciones/ } \\
\text { muerte }\end{array}$ & No & TB diseminada & No & No & No & No & No & No & No \\
\hline
\end{tabular}

AR: artritis reumatoidea; EC: enfermedad de Crohn; EA: espondilitis anquilosante; NR: no realizado; NC: no corresponde; INH: isoniazida.

ción de TB en pacientes que habían recibido tratamiento de ITBL plantea la importancia de un correcto estudio y tratamiento de ITBL, así como el seguimiento de los pacientes que reciben anti-TNF $\alpha$ para detectar en forma precoz esta complicación.

Si bien las alteraciones inmunológicas que producen estos fármacos constituyen el principal factor causal, existen otros potenciales como la epidemiología de la TB de cada país, el tipo de fármaco utilizado, la enfermedad de base y el cribado de ITBL realizado previo al inicio del tratamiento ${ }^{(8-10)}$.

En cuanto al factor epidemiológico, Uruguay actualmente pertenece al grupo de países con incidencia mediana-alta de TB (20-50 casos/100.000 habitantes) ${ }^{(11)}$, lo cual es de esperar que influya en la incidencia elevada encontrada en el grupo de pacientes con anti-TNF $\alpha$. Los estudios que han publicado datos de diferentes poblaciones provienen mayoritariamente de países con bajas tasas de incidencia de TB, lo cual constituye una limitante a la hora de comparar nuestros resultados ${ }^{(12,13)}$. En España, país que en el año 2004 presentaba una incidencia similar a la nuestra $(25 / 100.000)$, se encontró una incidencia de 514/100.00 para el grupo de pacientes con anti-TNF $\alpha$. Estas cifras descendieron significativamente luego de aplicar normativas que incluyeron el cribado de ITBL en forma obligatoria ${ }^{(8)}$.

En relación con los fármacos, la totalidad de los casos de TB se dieron en pacientes bajo tratamiento con adalimumab; esto es acorde a lo reportado a nivel inter- nacional, donde el mayor riesgo de TB se observó con anti-TNF $\alpha$ de tipo monoclonal versus etanercept ${ }^{(6,14)}$. Probablemente esta diferencia se relacione con el hecho de que adalimumab e infliximab bloqueen el TNF $\alpha$ soluble (TNFR1).

En cuanto a la enfermedad de base, dentro de las EI, la AR ha demostrado asociarse a mayor riesgo de $\mathrm{TB}^{(15)}$; si bien existe una predominancia de esta enfermedad en el grupo estudiado (6 de 9), el número total de casos registrados (9) impide establecer una asociación de esta EI con la TB.

Por último, en cuanto al cribado de ITBL y su tratamiento previo al inicio de los anti-TNF $\alpha$, varios países han elaborado guías o recomendaciones que, si bien plantean diferentes algoritmos de estudio, coinciden en la necesidad de su aplicación sistemática como principal estrategia para disminuir el riesgo de TB en este grupo de pacientes ${ }^{(8,16-18)}$. Los algoritmos propuestos insisten en la importancia de descartar TB, investigar exposición previa y realizar PPD o IGRA. En relación con priorizar uno u otro test, la mayoría de los autores recomiendan realizar ambos, considerando que el resultado positivo de cualquiera de ellos permite plantear ITBL y realizar el tratamiento ${ }^{(5,19-22)}$. Por otro lado, el resultado negativo de ambos tests permite en principio descartar ITBL. En la población estudiada, el cribado para ITBL fue heterogéneo y en la mayoría de los casos (8/1) se realizó únicamente una de las dos pruebas; teniendo en cuenta la dificultad en la accesibilidad a los IGRA en nuestro país, 
por no constituir una prestación obligatoria de los prestadores de salud, y la dificultad que ha ocurrido por períodos en la disponibilidad de la prueba de PPD.

El presente trabajo significó un cambio en el algoritmo de screening de los pacientes que van a recibir anti-TNF $\alpha$ en Uruguay, siendo requisito por normativa del FNR la realización de ambas pruebas, acorde a lo recomendado a nivel internacional ${ }^{(16)}$.

En relación con el tratamiento de la ITBL, si bien hay varios posibles planes $^{(23)}$, la mayoría de las guías coinciden en indicar INH por un período de nueve meses y es la pauta de elección en Uruguay. En la serie estudiada, cuatro de los casos habían recibido INH previamente, aun así desarrollaron TB. Esto podría deberse a dos factores. Por un lado, al breve período de tratamiento, seis meses y no nueve como lo recomendado. Por otro lado, se describe en la literatura que los pacientes que reciben anti-TNF $\alpha$ mantienen un riesgo elevado de desarrollar TB que requiere seguimiento estrecho para su detección $\operatorname{precoz}^{(7,8,10)}$.

En cuanto al tiempo de aparición de los casos de TB luego de iniciado el fármaco, se observaron casos de aparición precoz y casos de aparición tardía. Esto no coincide con los datos publicados por la mayoría de los autores, que señalan al primer año de exposición al fármaco como el período de mayor riesgo ${ }^{(24)}$. Sin embargo, hay otros autores que han encontrado un riesgo persistente de TB a lo largo del tratamiento con los agentes anti-TNF $\alpha$, señalando la importancia de la vigilancia a largo plazo ${ }^{(8)}$. Una posible explicación sería la relación de los casos precoces $(\geq 15$ meses) con una reactivación de una ITBL no detectada o insuficientemente tratada (casos 1, 2, 6, 8 y 9) y los casos tardíos (casos 3, 4 y 5) tratarse de una exposición nueva a M. tuberculosis (tabla 4).

Predominaron las formas de TB extrapulmonares o diseminadas, al igual que lo que sucede en otras poblaciones con inmunidad vulnerable, como es el caso de los coinfectados VIH. Sin embargo, a pesar de tratarse de un grupo de riesgo, no se registraron muertes.

\section{Conclusiones}

Encontramos una alta incidencia de TB en la población bajo cobertura con anti-TNF $\alpha$, siendo 16 veces mayor que en la población general. La presentación temporal de los casos fue variada y se dieron casos en sujetos que habían recibido tratamiento de ITBL previo, sugiriendo que el riesgo persiste mientras exista exposición al fármaco. Por todo esto es de suma importancia realizar cribado de ITBL previo al inicio de los mismos, y mantener siempre un alto índice de sospecha frente a cuadros compatibles.

\section{Agradecimientos}

A la Comisión Honoraria de Lucha Antituberculosa y Enfermedades Prevalentes y al Fondo Nacional de Recursos por su invaluable colaboración facilitando la información de sus registros de datos.

\section{Abstract}

Introduction: tuberculosis (TB) is a frequent complication in patients receiving tumor necrosis factor-alpha $(\mathrm{TNF}-\alpha)$ blockers. It occurs upon the reactivation of a latent infection or the progression of a recent infection.

Objective: to learn about the incidence of TB in a population receiving tumor necrosis factor-alpha $(\mathrm{TNF}-\alpha)$ blockers, to analyze the presentation of this condition and to conduct a latent infection research prior to the initiation of therapy.

Method: retrospective cohort study. Patients receiving tumor necrosis factor-alpha (TNF- $\alpha$ ) blockers between 2010 and 2016 were included in the study. Data were obtained from the IT systems of the National Resources Fund and the National Tuberculosis Program. The incidence of TB was calculated and the cases developing TB were described.

Results: 991 treatments were included for $980 \mathrm{pa}-$ tients in total. 9 cases of TB were reported. Global incidence was 419.9 (IC 95\% 191.9-591.2) out of 100,000 people per year. Cases of TB were only seen in patients treated with adalimumab. Screening for LTBI upon initiation of the drug was heterogeneous and the disseminated forms of TB prevailed (6/9) over isolated pulmonary affectation (3/9). In all cases anti-TNF $\alpha$ was suspended when TB was diagnosed, and it was not reinitiated.

Conclusions: the incidence of TB in patients receiving tumor necrosis factor-alpha (TNF- $\alpha$ ) blockers was 16.5 times greater than in the general population. Disseminated forms of TB prevailed, and some cases occurred in individuals who had received LTBI therapy prior to the initiation of the drug, suggesting the risk persists as long as there is exposure to the drug.

\section{Resumo}

Introdução: a tuberculose (TB) é uma complicação frequente do uso de fármacos anti-TNF $\alpha$. É causada pela reativação de uma infecção latente ou pela evolução de uma infecção recente.

Objetivos: conhecer a incidência de TB na população que recebeu fármacos anti-TNF $\alpha$, analisar as formas de apresentação e a realização de pesquisa de infecção latente prévia ao início do tratamento. 
Método: estudo de coorte retrospectivo. Foram incluídos todos os pacientes que receberam fármacos anti-TNF $\alpha$ no período 2010-2016. Os dados foram obtidos dos registros informatizados do Fondo Nacional de Recursos e do Programa Nacional de Tuberculosis. A incidência de TB foi calculada e foram descritos os casos que desenvolveram esta patologia.

Resultados: foram incluídos 991 tratamentos para um total de 980 pacientes. Nove casos de TB foram registrados. A incidência global foi de 419,9 (IC 95\% 191,9-591,2) por 100.000 pessoas/ano. Somente foram registrados casos de TB em pacientes tratados com adalimumab.

A triagem de infecção tuberculosa latente (ITBL) prévia ao início do fármaco foi heterogênea e predominaram as formas de TB disseminadas (6/9) sobre a pulmonar isolada (3/9). Em todos os casos o uso de anti-TNF $\alpha$ foi suspenso definitivamente quando o diagnóstico de TB foi realizado.

Conclusões: a incidência de TB na população de pacientes em tratamento com anti-TNF $\alpha$ foi 16,5 vezes maior que na população em geral. Predominaram as formas disseminadas de TB e foram registrados casos em pacientes que haviam recebido tratamento para ITBL prévio ao início do fármaco, sugerindo que o risco persiste enquanto houver exposição a este.

\section{Bibliografía}

1. World Health Organization. Global tuberculosis report 2016. Geneva: WHO, 2016:1-214.

2. Farga V, Caminero JA. Tuberculosis. 3ra ed. Santiago de Chile: Mediterráneo, 2011:474 p.

3. Lewinsohn DM, Leonard MK, LoBue PA, Cohn DL, Daley CL, Desmond E, et al. Official American Thoracic Society/Infectious Diseases Society of America/Centers for disease control and prevention clinical practice guidelines: diagnosis of tuberculosis in adults and children. Clin Infect Dis 2017; 64:111-5.

4. Horsburgh CR Jr. Priorities for the treatment of latent tuberculosis infection in the United States. N Engl J Med 2004; 350:2060-7.

5. Diel R, Goletti D, Ferrara G, Bothamley G, Cirillo D, Kampmann B, et al. Interferon-? release assays for the diagnosis of latent Mycobacterium tuberculosis infection: a systematic review and meta-analysis. Eur Resp J 2011; 37(1): 88-99.

6. Tubach F, Salmon D, Ravaud P, Allanore Y, Goupille P, Bréban M, et al. Risk of tuberculosis is higher with anti-tumor necrosis factor monoclonal antibody therapy than with soluble tumor necrosis factor receptor therapy. The three year prospective French Research Axed on Tolerance of Biotherapies registry. Arthritis Rheum 2009; 60:1884-94.

7. Comisión Honoraria de Lucha Antituberculosa y Enfermedades Prevalentes (CHLA-EP), Facultad de Medici-
na-UdelaR, Cátedra de Neumología, Cátedra de Enfermedades Infecciosas. Guía nacional para el manejo de la tuberculosis. $3^{\mathrm{a}}$ ed Montevideo: MSP, 2016:95 p.

8. Carmona L, Gómez-Reino JJ, Rodríguez-Valverde V, Montero D, Pascual-Gómez E, Mola EM, et al. Effectiveness of recommendations to prevent reactivation of latent tuberculosis infection in patients treated with tumor necrosis factor antagonists. Arthritis Rheum 2005; 52(6):1766-72.

9. Zhang Z, Fan W, Yang G, Xu Z, Wang J, Cheng Q, et al. Risk of tuberculosis in patients treated with TNF-á antagonists?: a systematic review and meta-analysis of randomised controlled trials. BMJ Open 2017; 7(3):e012567.

10. Organización Mundial de Salud. Informe mundial sobre la tuberculosis 2015. Sinopsis. OMS, 2015:5p.

11. World Health Organization. Global tuberculosis report 2018. Geneva: WHO, 2018.

12. Askling J, Fored CM, Brandt L, Baecklund E, Bertilsson L, Geborek P, et al. Risk and case characteristics of tuberculosis in rheumatoid arthritis associated with tumor necrosis factor antagonists in Sweden. Arthritis Rheum 2005; 52:1986-92.

13. Dixon WG, Hyrich KL, Watson KD, Lunt M, Galloway J, Ustianowski A, et al. Drug-specific risk of tuberculosis in patients with rheumatoid arthritis treated with anti-TNF therapy: results from the British Society for Rheumatology Biologics Register (BSRBR). Ann Rheumat Dis 2010; 69(3):522-8

14. Solovic I, Sester M, Gomez-Reino JJ, Rieder HL, Ehlers S, Milburn HJ, et al. The risk of tuberculosis related to tumour necrosis factor antagonist therapies: a TBNET consensus statement. Eur Resp J 2010; 36(5):1185-206.

15. Goletti D, Petrone L, Ippolito G, Niccoli L, Nannini C, Cantini F. Preventive therapy for tuberculosis in rheumatological patients undergoing therapy with biological drugs. Expert Rev Anti Infect Ther 2018; 16(6):501-12.

16. Pérez Sartori G. Tuberculosis en pacientes inmunodeprimidos no VIH: enfermedad autoinmune, reumatológica e inflamatoria intestinal. En: Comisión Honoraria de Lucha Antituberculosa y Enfermedades Prevalentes (CHLA-EP), Facultad de Medicina-UdelaR. Guía nacional para el manejo de la tuberculosis en situaciones especiales, año 2017. Montevideo: MSP, 2017:79-87.

17. Mir Viladrich I, Daudén Tello E, Solano-López G, López-Longo FJ, Taxonera Samso C, Sánchez Martínez P, et al. Consensus document on prevention and treatment of tuberculosis in patients for biological treatment. Arch Bronconeumol 2016; 52(1):36-45.

18. Tomio J, Yamana H, Matsui H, Yamashita H, Yoshiyama T, Yasunaga H. Tuberculosis screening prior to anti-tumor necrosis factor therapy among patients with immune-mediated inflammatory diseases in Japan: a longitudinal study using a large-scale health insurance claims database. Int J Rheum Dis 2017; 2(11):1674-83.

19. Bartalesi F, Vicidomini S, Goletti D, Fiorelli C, Fiori G, Melchiorre D, et al. QuantiFERON-TB Gold and the TST 
are both useful for latent tuberculosis infection screening in autoimmune diseases. Eur Resp J 2009; 33:586-93.

20. Lee SH. Diagnosis and treatment of latent tuberculosis infection. Tuberc Resp Dis (Seoul) 2015; 78(2):56-63.

21. Arias Guillén M. Avances en el diagnóstico de la infección tuberculosa. Arch Bronconeumol 2011; 47(10):521-30.

22. Freund R, Granger B, Francois C, Carcelain G, Ravaud P, Mariette X, et al. Cost-effectiveness analysis of strategies using new immunological diagnostic tests of latent tuberculo- sis infection before TNF-blockers therapy. Presse Med 2018; 47:e9-e13.

23. World Health Organization. Latent tuberculosis infection: updated and consolidated guidelines for programmatic management. Geneva: WHO, 2018:74 p.

24. Xie X, Li F, Chen JW, Wang J. Risk of tuberculosis infection in anti-TNF-á biological therapy: from bench to bedside. J Microbiol Immunol Infect 2014; 47(4):268-74.

\section{Contribución de autores}

Ana Gruss, https://orcid.org/0000-0002-9616-7985. Concepción, recolección de datos, diseño, análisis, interpretación de los resultados, redacción.

Mariela Contrera, https://orcid.org/0000-0002-5178-0807. Concepción, recolección de datos, diseño, análisis, interpretación de los resultados, redacción.

Natalia Piñeiro, https://orcid.org/0000-0002-5238-4494. Recolección de datos, análisis, interpretación de los resultados.

Abayubá Perna, https://orcid.org/0000-0001-7062-1310. Recolección de datos, análisis, interpretación de los resultados.

Rosana Gambogi, https://orcid.org/0000-0003-0781-7745. Análisis, revisión crítica.

Alicia Alemán, https://orcid.org/0000-0002-9247-1031. Análisis, revisión crítica.

Fernando Correa, https://orcid.org/0000-0001-8403-052X. Análisis, revisión crítica.

Henri Albornoz, https://orcid.org/0000-0001-7559-6193. Análisis, revisión crítica. 